山्山FRANÇAISE

$3 \mathrm{DE}$

夏PÉDAGOGIE

\section{Revue française de pédagogie}

Recherches en éducation

$195 \mid 2016$

Varia

\title{
Des « héritières » dans la formation d'assistante de service social ? Aspiration au travail social et reclassement
}

The "inheritor" women in the social work training? Educational ambition and

reclassement

\section{Ruggero lori}

\section{OpenEdition}

Journals

Édition électronique

URL : http://journals.openedition.org/rfp/5026

DOI : $10.4000 /$ rfp.5026

ISSN : 2105-2913

Éditeur

ENS Éditions

Édition imprimée

Date de publication : 30 juin 2016

Pagination : 37-50

ISSN : 0556-7807

Référence électronique

Ruggero lori, « Des « héritières » dans la formation d'assistante de service social ? Aspiration au travail social et reclassement », Revue française de pédagogie [En ligne], 195|2016, mis en ligne le 30 juin 2019, consulté le 05 janvier 2021. URL : http://journals.openedition.org/rfp/5026 ; DOl : https:// doi.org/10.4000/rfp.5026 


\title{
Des " héritières » dans la formation d'assistante de service social? Aspiration au travail social et reclassement
}

\author{
Ruggero lori
}

\begin{abstract}
Cet article interroge le choix par des étudiantes issues des classes supérieures de filières courtes et professionnelles pour devenir assistantes de service social. En partant d'une histoire de cette formation complétée par des données socio-démographiques générales, il resitue d'abord cette filière au sein de l'espace du supérieur. L'attention portée aux pratiques de recrutement dans ce segment professionnel permet ensuite de mettre en relation les dispositions particulières requises par les écoles lors de la sélection avec les appartenances de classes des étudiantes entrantes. Le regard plus particulier porté sur les étudiantes issues des classes supérieures permet de voir comment, loin de représenter des parcours linéaires, leurs trajectoires doivent être comprises à l'articulation des parcours antérieurs, des socialisations étudiantes diverses et des stratégies de recrutement des écoles.
\end{abstract}

Mots-clés (TESE) : enseignement supérieur, classe sociale, sexe, choix des études, orientation professionnelle, procédure de recrutement.

\section{Introduction}

La formation professionnelle au métier d'assistante de service social (ou plus fréquemment «assistante sociale »') a regroupé pendant longtemps et en large

1 Étant donné le faible pourcentage d'hommes dans cette formation ( $6 \%$ des nouveaux inscrits en 2013; Direction de la recherche, majorité des femmes célibataires des classes moyennes et supérieures, soucieuses de reconvertir, souvent contre l'avis parental, des expériences bénévoles et des dispositions d'« aide aux autres» acquises lors de leurs

des études, de l'évaluation et des statistiques [DREES], 2015) et dans notre échantillon, nous déclinerons les étudiants et le métier «au féminin » ou par sigle : ASS. 
trajectoires (Verdès-Leroux, 1978; Guerrand \& Rupp, 1978). Après plusieurs décennies d'«expansion scolaire» (Prost, 2013), ce cursus a progressivement évolué dans sa composition et regroupe aujourd'hui un public aux origines sociales fortement hétérogènes avec l'arrivée progressive d'étudiantes d'origines populaires, souvent jeunes bachelières. Elles représentent aujourd'hui environ la moitié de l'effectif'.

Un certain nombre de travaux français en sociologie de l'éducation (Bourdieu \& Passeron, 1964, 1970; Bourdieu \& de Saint-Martin, 1970 ; Convert, 2003, 2010) ont montré que le public qui s'oriente dans l'enseignement supérieur est fortement différencié selon les filières, au regard des propriétés genrées, scolaires, sociales ou encore territoriales (Nicourd, Samuel $\&$ Vilter, 2011). Les inégalités en la matière conditionnent largement la distribution des individus au sein de l'espace social que constituent les études supérieures.

Pour autant, si cette littérature scientifique nous renseigne sur les orientations des étudiants issus des classes populaires (Hugrée, 2010; Palheta, 2011; Orange, 2011; Pasquali, 2014; Truong, 2015) ou sur celles des étudiants du haut de la hiérarchie scolaire (Blanchard, 2012; Darmon, 2013), s'intéresser plus spécifiquement aux choix des élèves des classes supérieures dans des filières situées "en bas» de la hiérarchie scolaire et professionnelle est plus inédit. Une telle entrée permet d'éclairer plus spécifiquement les processus de déclassement et reclassement dans le supérieur. En s'éloignant de la rhétorique professionnelle qui souligne un fort engagement dans le métier ${ }^{3}$

2 Parmi les nouveaux entrants en 2014, selon la catégorie socioprofessionnelle du père, on retrouve $52,2 \%$ d'enfants dont le père est employé, ouvrier ou inactif, 10,8 \% dont le père exerce une profession intermédiaire, $23 \%$ d'enfants de pères cadres, 10,6 \% d'un père artisan, commerçant ou chef d'entreprise, 3,3\% ayant un père agriculteur. Du côté des mères, on retrouve $66 \%$ d'enfants dont la mère est employée, ouvrière ou n'a jamais travaillé, 16,2 \% dont la mère exerce une profession intermédiaire, 11,4\% d'enfants de mères cadres, 4,9\% d'une mère artisane, commerçante ou chef d'entreprise, 1,5\% d'une mère agricultrice (DREES, 2016).

3 L'assistante sociale est souvent présentée par les acteurs politiques ou les centres de formation comme faisant partie des métiers qui incluent un aspect "vocationnel», où "on n'arrive pas par hasard», et pour lequel être dotée d'une "fibre militante» peut s'avérer «un plus» (lori \& Nicourd, 2014). C'est le cas pendant l'année 2015 du Premier ministre Manuel Valls et de la Secrétaire d'État chargée des personnes handicapées et de la lutte contre l'exclusion qui soulignent, dans leurs déclarations respectives, la "passion», la «conviction», l'«engagement» et le fait "qu'on ne devient pas travailleur social par hasard», participant par là à naturaliser le par- ou encore, de manière cyclique, un «déclin de vocation» (Molina, 2016), il s'agit, dans cet article ${ }^{4}$, d'interroger les choix scolaires et les orientations professionnelles des étudiantes assistantes sociales, en analysant des mobilités sociales en train de se faire (Pagis \& Pasquali, 2016). Alors que ces formations se destinent et attirent essentiellement des jeunes filles issues des classes moyennes et populaires, nous mettrons ainsi en évidence, à travers le cas des étudiantes de classes supérieures qui s'y engagent, les raisons de ces orientations a priori «improbables» (Lahire, 1994; Mercklé, 2005). En quoi les dynamiques de déclassement (Peugny, 2007; Eckert, 2014) contribuent-elles à expliquer l'orientation de ces individus vers ces filières? Peut-on réduire les parcours de ces étudiantes à des «trajectoires interrompues», à des transmissions familiales dysfonctionnelles (Henri-Panabière, 2010) et à des décalages ou reconversions "faute de capital social» (Bourdieu, 1978, p. 4) dans des métiers moins qualifiés que ceux qui les «attendaient » à la sortie du système scolaire secondaire? Ces scolarités sont-elles, de fait, atypiques pour ces filières?

Pour répondre à cet ensemble de questions, nous partons du présupposé que l'orientation dans l'enseignement supérieur est autant tributaire des trajectoires antérieures des intéressées que de socialisations étudiantes diverses et des stratégies de recrutement à l'œuvre au sein des écoles. Dès lors, une première partie de cet article vise à faire une brève présentation de I'histoire de cette filière professionnelle, complétée par des données socio-démographiques générales permettant de la resituer au sein de l'espace du supérieur. De là se dessineront trois profils d'orientation dans cette filière. En second lieu, on s'intéressera aux pratiques de recrutement des centres de formation, pour voir comment les stratégies institutionnelles sont basées sur des compétences socialement situées et sont aujourd'hui remises en cause par les changements du public à l'entrée. En dernière partie, nous questionnerons les caractéristiques de ces jeunes femmes issues des classes supérieures pour montrer comment, loin de dépendre de parcours linéaires $d^{\prime}$ ' héritières », leurs orientations doivent être comprises au croisement de recompositions d'héritages familiaux singuliers et des changements de l'offre scolaire et professionnelle.

4 Je tiens particulièrement à remercier Julie Minoc, Florence Eloy et Sandrine Nicourd pour leurs relectures et conseils avisés, ainsi que les deux relecteurs anonymes et les rédacteurs en chef de la revue pour leurs commentaires. 
Cet article repose sur un travail doctoral en cours portant sur la formation des ASS en France et en Italie. En complément de statistiques publiques sur les étudiants entrant dans des formations sociales en France (DREES 2000-2016), il s'appuie principalement sur des données originales issues d'une enquête qualitative et quantitative de suivi de cohortes débutée en 2013 (enquête «FRITA», 2013-2016) auprès de deux formations d'assistante sociale en région parisienne, l'une en école spécialisée et l'autre en institut universitaire technologique (IUT, diplôme universitaire de technologie "carrières sociales"). Au total, 190 individus composent l'échantillon (dont 82 ont été interrogés uniquement en première année et 108 réinterrogés à chaque début d'année jusqu'à l'obtention du diplôme). À partir des réponses issues du premier questionnaire (axé sur les origines scolaires et sociales, sur les expériences précédentes et sur les aspirations et projets à l'entrée), nous avons ensuite procédé à une analyse de correspondance (ACM) et à une classification ascendante hiérarchique $(\mathrm{CAH})$ produites par le logiciel RStudio, qui nous ont permis de distinguer trois modalités d'entrée dans cette filière. Parallèlement, des entretiens ont été menés au début de chaque année de formation avec une partie de ces étudiants (pour un total de 37 entretiens avec 16 étudiants tout au long des trois ans); il s'est agi d'interroger plus spécifiquement leurs parcours de vie et conditions de socialisation, leurs histoires familiales et scolaires, leurs différents choix d'orientation, leurs motivations à entrer dans ce cursus et le déroulement de leurs études jusqu'au moment du diplôme. Au sein de la population enquêtée, les étudiants des «classes supérieures » (fils et filles d'un père ou mère «cadre ou profession intellectuelle supérieure») représentent $28 \%$ des individus de notre échantillon (49 individus au total, 23 interrogés quantitativement uniquement en première année, 26 suivis lors des trois ans, dont 8 par entretien). C'est sur cet échantillon en particulier que se base l'essentiel des analyses présentées ici. Par ailleurs, nous avons mené des observations tout au long des trois ans du cursus, lors de différents moments de la formation : entretiens de sélection, réunions de pré-rentrée, cours et séminaires, réunions pédagogiques, etc. Ces observations ont été complétées par des entretiens formels et informels avec différents acteurs de la formation : du formateur responsable pédagogique aux enseignants, des vacataires aux «personnalités» historiques du champ.

\section{Une formation centenaire à la croisée des filières du supérieur}

De manière concomitante à la structuration d'un État social naissant et à l'instar des études d'infirmière («visiteuse»), l'encadrement de la formation d'assistante sociale - figure «canonique» des métiers du travail social - se matérialise en 1938 avec la création d'un diplôme d'État. Au départ, le public de cette formation est uniquement composé par des femmes des couches supérieures, ne trouvant pas d'autres possibilités d'accès aux filières universitaires. Initialement basée sur des savoirs peu formalisés empruntés aux sciences humaines et sociales et aux disciplines médicales, cette formation se transforme dans l'après-guerre avec l'introduction et le développement de méthodes professionnelles spécifiques (étude de cas individuels et travail collectif). C'est lors de cette période de démocratisation relative du recrutement que le métier se rapproche de ce qu'on observe aujourd'hui et que ses effectifs augmentent : le corps professionnel change dans sa composition et voit l'arrivée d'un public «moyen » issu dans une moindre proportion d'origines sociales élevées, et plus jeune, permettant à de nombreuses femmes (et à une minorité d'hommes) en réo- rientation une possibilité d'ascension sociale ${ }^{5}$. Parallèlement, en dépit des changements structurels dans l'offre du supérieur, les filles des classes supérieures, plus dotées socialement et scolairement et qui caractérisaient la majorité du métier dans les années 1930 et 1940, sont orientées vers d'autres voies symboliquement plus réputées ${ }^{6}$. La profession d'assistante sociale

5 L'étude de Simone Crapuchet (1987) atteste que la proportion d'enfants de classes supérieures commence à s'effondrer à partir de la Libération, jusqu'à représenter une «minorité témoin » dans les années 1960, et qu'inversement la proportion de filles des cadres moyens et d'employés s'accroît jusqu'à représenter $3 / 5^{e}$ des effectifs étudiants en 1976. L'étude de I'INSERM (Brams \& Courtecuisse, 1972) sur les étudiants entrants en 1970 souligne également une «moyennisation » plus importante : $27 \%$ des étudiants sont issus d'un père exerçant une profession de cadre supérieur ou de profession libérale, $41 \%$ d'un père cadre moyen, cultivateur, commerçant ou artisan, et $29 \%$ d'un père employé ou ouvrier. Francine Muel-Dreyfus dans son étude sur les éducateurs de la période 1969-1975 (groupe professionnel proche des ASS) enregistre des chiffres similaires : $16 \%$ issus des classes supérieures, $46 \%$ des classes moyennes, $9 \%$ d'exploitants agricoles, et $29 \%$ de la classe ouvrière (Muel-Dreyfus, 1983).

6 Concernant les stratégies de reclassement des filles des classes supérieures, on peut citer notamment le travail de Vincent Dubois qui observe une élitisation et une féminisation du recrutement dans les formations du domaine culturel et qui repère des stratégies vocationnelles visant davantage le secteur (culturel) que les métiers de ce secteur en tant que tels (Dubois, 2013). 
se «fonctionnarise » quant à elle et sa formation, organisée dans des centres de formation agréés par le ministère des Affaires sociales, continue à produire un diplôme court et professionnel' ${ }^{7}$, en parallèle des études plus académiques.

En effet, contrairement à d'autres pays où la formation en travail social a été intégrée à l'université (Canada, Espagne, Italie, entre autres) ou à d'autres cursus proches (Instituts de formation en soins infirmiers ou sciences de l'éducation), le système de formation français conserve une certaine autonomie dans l'espace de l'enseignement supérieur (Iori, 2017). Pourtant, et alors même que les responsables de la formation revendiquent leur distance à la culture universitaire et des formes de contestation du modèle scolaire $^{8}$, la réforme des années 1980 tente bien des rapprochements avec les études universitaires (introduction d'un mémoire de recherche, place des sciences humaines et sociales et en particulier de la psychologie, dans la formation; Delaunay, 2006). Comme d'autres secteurs de l'administration publique (Ropé \& Tanguy, 1994; Agulhon, 2005; Neyrat, 2008) et dans la lignée des réformes européennes sur la formation « tout au long de la vie» (Charle \& Soulié, 2008), le cursus est restructuré en 2004 selon la logique des «compétences » qui - concomitamment d'ailleurs aux entreprises d'individualisation de la question sociale (Astier, 2009) que ces futures assistantes sociales seront chargées d'appliquer - étend l'individualisation du parcours de formation. Cette nouvelle réforme (révisée en 2008) ancre l'apprentissage sur des «référentiels de compétences » à acquérir tout au long des trois années de formation. Si cela introduit certes des passerelles avec l'université (elle-même soumise aux mêmes réformes libérales qui contribuent à en changer la mission), la formation garde pour l'instant son autonomie (relative) et assoit son aspect pleinement professionnalisant. Bien que d'autres réformes aient tenté plus récemment une réorganisation structurelle ${ }^{9}$, cette

7 Selon le niveau reconnu par le système éducatif supérieur français, les formations "courtes» ont une durée de trois ans, mais, de par leur caractère professionnel, elles ont un niveau «bac +2 », différent donc du niveau d'une licence universitaire de trois ans après le baccalauréat (bac +3 )

8 On retrouve également cette distanciation par rapport aux savoirs scolaires et la mise en valeur d'un savoir «de terrain» dans les discours des travailleurs sociaux belges étudiés par Jean-François Gaspar (2007).

9 Depuis 2011 se succèdent des projets de réforme, entre uniformisation européenne et nivellement des différentes formations formation reste gérée et organisée dans des écoles privées ou publiques ou semi-publiques/associatives, sous tutelle de l'État ${ }^{10}$.

Cependant, toutes les écoles ne regroupent par les mêmes profils d'élèves. Le numerus clausus adopté par chaque école est lié à l'attribution des fonds publics par chaque région (compétente en matière de formation professionnelle) à ces instituts. L'espace des écoles de travail social doit alors être considéré, tant au niveau national que régional, comme un espace concurrentiel où les étudiantes se distribuent différemment d'un centre de formation à l'autre. En effet, chaque école porte une histoire et une idéologie particulières, certaines proposent un double cursus avec l'universitél1, ou encore ciblent des profils spécifiques d'élèves. Dès lors, on ne retrouve pas le même type de public, en termes d'origines sociales ou scolaires, dans une école centenaire parisienne ou dans un IUT dispensant un diplôme de carrières sociales ${ }^{12}$.

En outre, contrairement à la plupart des filières post-bac (université, sections de techniciens supérieurs préparant aux BTS, classes préparatoires aux grandes écoles, diplômes universitaires de technologie, première année commune aux études de santé, entre autres), ces cursus en travail social (à l'exception des formations en IUT) sont absents des choix accessibles dans I'application Admission Post-Bac (APB),

tion de Hautes Écoles spécialisées (selon le modèle suisse, belge ou celui des écoles de santé françaises) et la mise en place d'un "tronc commun » aux formations de niveau III (assistantes sociales, conseillères en économie sociale et familiale [CESF], éducateurs spécialisés [ES] et éducateurs de jeunes enfants [EJE]) ont été envisagées lors des différences négociations entre les acteurs du travail social et les pouvoirs publics.

10 Dépendantes de la Direction régionale de la Jeunesse, des Sports et de la Cohésion sociale (DRJSCS) et hors Éducation nationale.

11 Ces doubles cursus se sont développés depuis quelques années au sein de certaines écoles et permettent d'atteindre, au bout de trois ans de formation, une certification de niveau bac +3 , en validant quelques examens universitaires et un mémoire conjoint école/ université.

12 Le centre de formation régional enquêté est une des premières écoles de service social en France, il propose un parcours d'ASS en double cursus avec une licence francilienne en sciences de l'éducation et la promotion est très large (environ 70-80 étudiantes). L'IUT assure au contraire ces formations depuis deux décennies. Il propose également, à côté de l'accompagnement au diplôme d'État d'ASS, une licence professionnelle et un diplôme universitaire de technologie en carrières sociales et c'est le seul type de formation en travail social recensé dans le classement de l'application Admission Post-Bac (APB). Ici, le recrutement est davantage ciblé, en favorisant par des points supplémentaires dans la sélection des élèves issus du même département, souvent plus «populaires » et plus jeunes par rapport à la population générale des écoles de travail social. 
dispositif censé classer les « vœux» des bacheliers dans le supérieur. De la sorte, ces filières conservent majoritairement leur propre calendrier de recrutement et leur aspect sélectif, en dehors de l'Éducation nationale. L'inscription dans cette filière suppose par conséquent un investissement non seulement personnel mais financier important : l'entrée dans ce cursus peut prendre plusieurs années, se concrétiser après plusieurs tentatives et échecs au concours, ou encore après une inscription dans des "prépas sociales »" épreuves de sélection sont payantes; certaines écoles ont par ailleurs des droits d'inscription plus élevés qu'à I'université, etc. Le fait d'être autonome ou de ne pas avoir de «soucis financiers» demeure d'ailleurs un critère demandé par ces écoles pour «tenir» durant ces trois années. Dès lors, l'entrée dans l'école se fait à des âges plus avancés que dans d'autres filières post-bac et souvent pour des candidates qui ont déjà eu au moins une expérience professionnelle, ce qui élargit I'hétérogénéité de son public.

Parallèlement aux enquêtes diffusées dans le monde universitaire, journalistique, ou encore professionnel, illustrant les conditions de vie et d'emploi précaires ${ }^{14}$, plusieurs instituts de formation soulignent des difficultés dans le remplissage des promotions (Union nationale des associations de formation et de recherche en intervention sociale [UNAFORIS], 2014) selon les «standards» en vigueur ${ }^{15}$. Pourtant, environ 10000 candidates se sont inscrites aux épreuves de sélection de l'ensemble des écoles et instituts en 2013 et seulement 3000 d'entre elles y ont été admises. Et si l'on s'intéresse aux statuts des entrantes en 2013 (DREES, 2015), les étudiantes demeurent certes la majorité $(70 \%)$, mais la multiplication d'autres types de profils est notable : des demandeuses d'emploi (25\%),

13 Ces «prépas» sont des formations payantes de quelques mois, souvent communes aux différents métiers du secteur social de niveau III, préparant aux concours d'entrée et permettant d'effectuer une première expérience de stage dans le secteur. Elles sont organisées dans les écoles sociales ou dans le premier cycle de certains cursus universitaires.

14 On fait référence ici à un ensemble d'études et de travaux soulignant la croissance des publics pris en charge, des dossiers, la flexibilité des horaires, la précarisation des emplois, ou encore le «burn-out» des travailleurs sociaux. À titre d'exemple, on peut citer la troisième partie de l'ouvrage La misère du monde dirigé par Pierre Bourdieu (1993), intitulée « Démission de l'État», qui a eu beaucoup de succès dans cet espace professionnel.

15 Ils parlent notamment d'une «baisse » du nombre et du niveau des candidats aux concours. Les données de la DREES enregistrent également une baisse de $4 \%$ du nombre des diplômés dans les formations de niveau III et de presque $10 \%$ pour le diplôme d'État d'ASS (DREES, 2016). des salariées ( $3 \%$ ) ou des agentes de la fonction publique ( $2 \%$ ) en congé de formation, ou encore des apprenties $(0,5 \%)$. Cela a pour corollaire des inscriptions plus ou moins tardives dans cette filière postbac : seul un quart des élèves inscrites en formation d'ASS en 2014-2015 a moins de 20 ans $^{16}$. D'ailleurs, si deux tiers des élèves ont le niveau bac, $30 \%$ ont déjà un autre diplôme supérieur ${ }^{17}$.

Prendre en considération ce positionnement «à part» de ces formations dans le supérieur se révèle un préalable nécessaire pour mieux comprendre la forte hétérogénéité que présente son public tout au long de l'histoire de la formation, tant sous le rapport de I'origine sociale que de l'âge. Quelles sont alors les conditions sociales et scolaires du choix d'orientation vers ces études professionnelles?

\section{Une sociographie des élèves en formation}

L'hétérogénéité des publics dans ces cursus témoigne de la diversité des trajectoires qui y mènent : des élèves d'origine populaire ayant choisi des bacs généraux ou technologiques et voulant continuer dans des filières professionnelles aux femmes d'origine sociale élevée en déclassement. Une comparaison avec les données de l'Observatoire de la vie étudiante (OVE, 2013) nous permet de mieux caractériser les origines sociales et scolaires des étudiantes suivant la formation d'ASS. Bien que composée majoritairement d'élèves aux pères employés et ouvriers, cette formation est moins «populaire » que les STS par exemple (Orange, 2011) où les bacheliers technologiques et professionnels constituent la majorité des promotions ( $81 \%$ contre $34 \%$ ). Les étudiantes de père "cadre» sont certes plus présentes dans cette filière (23\%) qu'au sein des STS (14\%), mais moins qu'à l'université (30\% toutes filières confondues) ou que dans les classes préparatoires aux grandes écoles (50\%).

Plusieurs sous-populations composent notre échantillon (voir tableau 1). La dispersion en termes d'origine sociale est patente et constitutive même de

$1625,7 \%$ ont moins de 20 ans, $34,4 \%$ entre 20 et 22 ans, $13,2 \%$ entre 23 et 25 ans, $10,3 \%$ entre 26 et 30 ans, et $16,5 \%$ plus de 30 ans (DREES, 2016)

17 Chez les élèves entrant en première année, 9,8 \% possèdent un diplôme de BTS, $10 \%$ une licence, 5,9 \% un DUT ou ont un niveau L2, 4,6\% ont déjà obtenu un Master 1 ou un Master 2 (DREES, 2016). 
Tableau 1. Milieu social d'appartenance selon la profession du père et de la mère (2013-2014)

\begin{tabular}{l|c|c}
\hline & N & $\%$ \\
\hline Milieu social favorisé & 10 & 5,2 \\
\hline $\begin{array}{l}\text { Milieu } \\
\text { social favorisé - intermédiaire }\end{array}$ & 21 & 11 \\
\hline Milieu social favorisé - populaire & 18 & 9,5 \\
\hline Milieu social intermédiaire & 38 & 20 \\
\hline Milieu social intermédiaire-populaire & 29 & 15,3 \\
\hline Milieu social populaire & 74 & 39 \\
\hline Ensemble & 190 & 100
\end{tabular}

Source : Enquête FRITA, 2013-2016.

Lecture : 10 étudiants, soit 5,2\% de l'ensemble, sont issus d'un milieu social favorisé, leurs deux parents appartenant à la catégorie des "cadres et professions intellectuelles supérieures».

ces cursus. La prise en compte de manière conjointe de la catégorie socio-professionnelle du père et de la mère nous aide à mieux visualiser les poids des différents milieux sociaux.

Dans notre échantillon, les femmes des «classes supérieures $»^{18}$ représentent environ $26 \%$ du public $(N=49)$. Elles sont plus souvent que le reste des élèves enquêtées passées par une scolarisation dans le privé (39\% contre $29 \%$ ) et davantage représentées dans la catégorie des bacheliers généraux (71 \% contre $56 \%$ ), un tiers d'entre elles étant passées par des filières non générales (21\% par un bac technologique, $8 \%$ par un bac professionnel). Alors que la proportion d'élèves ayant subi au moins un redoublement est élevée (48 \%) et que les mentions au bac sont rares dans l'ensemble de la population (40\%), les filles des classes supérieures sont davantage "à I'heure» au bac que leurs collègues (59\% contre $49 \%$ ) mais moins nombreuses à y obtenir une mention (33\% contre $39 \%$ des classes moyennes et $45 \%$ des classes populaires). Cela

18 On considère ici par filles des «classes supérieures» les élèves ayant un noyau familial composé d'un père ou d'une mère «cadre ou profession intellectuelle supérieure». Par conséquent, si dans certains cas, on est face à des familles de classes supérieures «homogames» (les deux membres faisant partie de ces catégories), dans la plupart des cas les origines familiales sont mixtes : supérieure/ moyenne ou supérieure/populaire; nous avons donc tenu compte tant des situations où le père est cadre et la mère employée que des situations (plus rares) où la mère occupe une profession libérale et le père une place d'ouvrier. Il est important à cet égard de considérer le rôle central de la mère dans la reproduction sociale (tant du point de vue de l'éducation, de la scolarisation que de l'orien- confirme plus largement les recherches sur les orientations dans le supérieur selon l'origine sociale, les étudiants des classes populaires qui continuent audelà du bac sont à la fois «sur-sélectionnés» scolairement, avec l'obtention de bons résultats, et «soussélectionnés» socialement, étant portés à choisir des filières courtes et professionnelles, contrairement à la plupart des étudiants d'origines supérieures (Hugrée \& Poullaouec, 2011).

Soulignons par ailleurs la particularité de ces formations du point de vue des écarts d'âge. Conformément à nos propres observations, depuis plusieurs années, les données de la DREES notent un progressif rajeunissement des nouvelles entrantes (10\% d'entre elles ont entre 18 et 19 ans en 2002, $26 \%$ aujourd'hui; DREES 2003-2015). Les étudiantes «jeunes» (entre 18 et 19 ans) représentent $31 \%$ des enquêtées de notre échantillon. Si l'on regarde ce qu'il en est spécifiquement du côté des filles des classes supérieures, elles demeurent plus âgées que la moyenne (23,7 ans à l'entrée en formation). Leur choix se fait moins immédiatement "après le bac» ou au cours des études secondaires, mais bien plutôt après d'autres expériences universitaires ou professionnelles (67\% contre $57 \%$ ).

La classification ascendante hiérarchique produite à partir des résultats d'une analyse factorielle ${ }^{19}$

19 Pour l'analyse factorielle, nous avons retenu 8 variables actives et 6 supplémentaires recodées à partir du premier questionnaire administré à l'entrée de la formation (classes d'âge en trois catégories, origine sociale en trois catégories, type de bac, diplôme des parents, type de public envisagé à la sortie - handicap, enfants... -, type de secteur - public, associatif, privé, polyvalence... -, projet 
confirme ces résultats et distingue trois groupes dans la population d'étudiantes assistantes sociales. Le plus nombreux ( $\mathrm{N}=71 ; 37 \%$ ) comprend avant tout des étudiantes ayant choisi de s'orienter dans ce cursus après d'autres expériences professionnelles ou formatives; elles ont, en revanche, rarement eu d'expériences d'«aide» au sens large (professionnelles ou bénévoles). Les étudiantes de ce premier groupe sont principalement âgées de 21 à 23 ans, ou, en moindre partie, de plus de 24 ans. S'il ne se caractérise pas par des différences de classe très marquées, c'est un groupe très hétérogène, les justifications en termes de "vocation» sont visibles mais l'accent est davantage mis sur l'aspect «pratique » de la formation et du métier (variété des publics et des débouchés à la sortie) et moins sur les «études». Il est composé en large partie par les étudiants de l'école régionale enquêtée qui s'opposent aux individus de la deuxième classe.

Celle-ci ( $N=64 ; 34 \%)$ est composée par les étudiantes plus «jeunes»(18-20 ans), ayant intégré ces formations à l'issue du bac, primo-arrivants dans le supérieur, souvent après une décision déjà prise dès la première. C'est un groupe marqué par une continuité d'études entre le secondaire et le supérieur; on y retrouve notamment les étudiantes ayant obtenu un bac technologique de santé et du social (ST2S), n'ayant pas eu auparavant d'expériences d'«aide». S'il n'inclut pas uniquement des étudiantes issues des classes populaires, on y retrouve des individus avec des parents n'ayant pas atteint le bac et plus «homogènes » à partir des caractéristiques d'âge.

Le dernier groupe $(\mathrm{N}=55 ; 29 \%)$ est celui qui nous intéresse plus particulièrement. C'est le seul groupe où les appartenances de classes ressortent de façon plus marquée, la majorité des étudiantes d'origine supérieure en fait partie. Ce groupe se distingue par un choix tardif pour cette formation après d'autres expériences formatives et professionnelles. L'intensité de la «vocation» y est la plus forte. Plus âgés en moyenne que ceux composant les autres groupes (24 ans ou plus), les individus de ce groupe sont en partie issus de filières générales (littéraire ou économique et social)

professionnel envisagé après les trois ans, élément le plus important dans le choix du métier, moment du choix du métier - avant le secondaire, avant le bac, après d'autres expériences -, expérience professionnelle dans la santé ou dans le social, engagement associatif, redoublement, type d'école, vocation dans le social), significatives du test de chi-deux au seuil de $5 \%$. La classification ascendante hiérarchique $(\mathrm{CAH})$ qui suit part de la même analyse factorielle. et ont eu majoritairement des expériences professionnelles ou bénévoles d'«aide».

Cette classification permet de voir le fort lien qui perdure entre les trajectoires scolaires et sociales d'une part, et des temporalités distinctes à l'entrée en formation d'autre part. Si l'origine sociale est un critère modérément distinctif dans la typologie présentée, elle réapparaît dans les représentations que ces étudiantes se font de leurs stratégies. Les étudiantes du dernier groupe en particulier rapportent davantage d'expériences « valorisantes » qui fondent leur choix et qui montrent qu'elles n'ont pas vécu dans la protection d'un parcours balisé par un «destin» social, comme c'est plutôt le cas pour les étudiantes de la seconde classe. Nous avons affaire ici à des étudiantes qui font face à un déclin social relatif dans leur trajectoire. Moins renfermées dans un objectif de court terme comme peut l'être celui d'un horizon professionnel à la sortie des trois ans, ces "héritières » sui generis réadaptent leurs expériences scolaires et professionnelles pour confirmer leur réorientation. Les écarts ainsi constatés avec le reste des étudiantes (en particulier avec celles d'origines populaires et des deux premiers groupes) reflètent en partie les différences de stratégies étudiantes présidant à l'orientation vers ces filières, davantage marquées par un choix pour des études supérieures et moins pour un travail d'assistante sociale en tant que tel. Mais qu'en est-il des stratégies institutionnelles mises en œuvre par les centres de formation concernant leur recrutement? En l'absence de données sur l'origine sociale des candidates aux épreuves de sélection, qui nous auraient permis de mieux savoir qui parvient ou échoue à intégrer ces filières (Vozari, 2014; Molina, 2016), il faut maintenant porter notre attention sur le processus de recrutement de ces étudiantes et étudier les stratégies spécifiques des deux écoles de notre échantillon.

\section{La sélection de compétences socialement situées}

En complément de «signes» vocationnels déjà observés par Romuald Bodin en ce qui concerne le travail de repérage de futurs éducateurs spécialisés (Bodin, 2009), le processus de sélection des écoles de travail social s'opère sur plusieurs mois et par étape. De I'envoi du dossier aux oraux en passant par les écrits, il suppose souvent une connaissance préalable du système de formation. Les dossiers en eux-mêmes 
peuvent faire l'objet d'une notation - qui diffère selon les écoles - visant notamment à évaluer les expériences «valorisantes» (travail associatif, voyages, militantisme, etc.). La validation d'une épreuve écrite (une dissertation ou un écrit portant sur des questions d'actualités, voire l'analyse d'un texte, devant permettre de mettre en valeur les capacités rédactionnelles ou de synthèse) permet l'accès à une (voire deux) épreuve(s) orale(s) devant des enseignants "formateurs», psychologues, professionnels, anciennes personnalités du secteur. Dans le cas d'une des écoles enquêtées, les formateurs valident le passage à la session orale après une discussion de groupe autour des écrits.

Concrètement, les diverses épreuves visent d'abord pour le jury de sélection à évaluer la capacité des candidats à s'inscrire dans le parcours de formation; elles privilégient de fait les étudiantes ayant déjà eu auparavant d'autres expériences professionnelles ou universitaires et celles qui soulignent leur « motivation» à entrer dans le secteur. Ainsi, le fait de faire preuve de «distance » dès le processus de sélection, de se montrer intéressé par l'actualité ou par les actualités du secteur professionnel, permet alors de convaincre les professionnels que I'on est apte à devenir ASS. C'est alors la mise en valeur, à tout moment - dans des lettres de motivations, dans les écrits comme à l'oral -, d'éléments susceptibles de concerner le «social» ou un «engagement» de l'élève qui va «faire la différence» : connaissance de l'actualité, expériences diverses dans l'associatif, possession du Brevet d'aptitude aux fonctions d'animateur en accueils collectifs de mineurs (BAFA), membre de son entourage familial exerçant dans le social (au sens large), capacités d'argumentation, etc. Ces éléments doivent alors être mobilisés par la candidate dans une reconstruction rétrospective de son propre parcours, de ses motivations, et seront repérés par les formateurs des écoles, dans l'idéal de «profils des personnes qui ont vraiment réfléchi à ce qu'ils font, des personnes pour lesquelles y a pas de problèmes sur le plan cognitif quoi, qui ont un bon niveau... qui savent élaborer $»^{20}$.

La sélection est alors centrée en large partie sur des dispositions «informelles». Les réquisits de l'institution - avoir du recul sur soi, ne pas être arrivé «par

20 Entretien avec Nicole, 55 ans, originaire d'une famille espagnole émigrée en France pendant les années 1930. ASS de formation, elle a travaillé en polyvalence et dans l'aide aux migrants pendant hasard » dans la formation - contribuent autant à susciter qu'à renforcer les discours vocationnels, à relire son passé en fonction d'un ethos professionnel singulier. Cette insistance sur la vocation, valorisée tant dans les discours des étudiantes que dans le travail des formateurs, participe à construire l'arrivée dans le social comme quelque chose de «naturel », à valoriser davantage ce que l'on "est» que ce que l'on "sait» (MuelDreyfus, 1983, p. 202). Cela peut s'inscrire, à l'instar du développement du projet personnel et professionnel en IUT où les étudiants sont amenés à expliciter leur «curiosité » et à être « responsables de soi-même», plus globalement dans les rhétoriques contemporaines ou «néo-libérales» demandant à l'étudiant d'être «entrepreneur de soi-même» (Tralongo, 2008, p. 102-103). En entretien avec les candidates, les formateurs consacrent l'arrivée de l'élève en formation comme la conséquence d'un engagement "personnel». Ils n'intègrent d'ailleurs que très peu dans leurs propres cours des approches en termes d'origine sociale, de modes de vie, de genre, de parcours et d'âges.

Or, les «bonnes» candidates recherchées répondent à des profils construits à l'image des travailleurs sociaux d'antan et rassemblant des dispositions davantage présentes chez un type particulier d'étudiantes. Comme l'indique une responsable de la sélection dans une école suivie: «Tu as des personnes qui sont dans des milieux tout à fait aisés, qui ont des conjoints qui ont des activités professionnelles qui leur permettent de subvenir à leurs besoins, tu vois vraiment, là pour le coup c'est vraiment un choix très déterminé... où y a pas d'enjeux financiers!». C'est notamment l'exemple d'Emma ${ }^{21}$, étudiante qui reçoit la note finale la plus élevée (18/20) parmi l'ensemble de celles de son année. Dans son dossier de recrutement, les formateurs notent ${ }^{22}$ :

Trajet scolaire et professionnel : [...] émergence de I'idée d'exercer en qualité d'ASS. Le domaine de la

21 Emma, 26 ans, bac ES «à I'heure» sans mention, licence en psychologie. Père médecin, mère secrétaire, elle vit son enfance chez ses parents jusqu'à leur divorce, pour ensuite vivre entre la maison de sa mère et celle des grands-parents paternels (grand-père militaire et grand-mère professeure universitaire des langues orientales). Suite à la mort de son père pendant son adolescence, elle s'installe chez sa mère où elle s'occupe de ses deux petites sœurs, jumelles, l'une inscrite dans des études de psychologie, l'autre intégrant la même année qu'Emma une formation d'éducatrice spécialisée après une licence de médiation culturelle.

22 Fiche d'évaluation d'Emma, manuscrite, rédigée par deux formateurs à la suite de son entretien, dernière session de sélection 2013. 
vente ne lui correspond pas ( $\neq$ éthique!). Anime des sites sur internet. Réside à Paris avec son fiancé (termine sa thèse, salarié), pas de soucis matériels.

Capacités cognitives : excellentes, elle adore lire et écrire (Le Monde, Courrier International, Causette, Littérature...) et adore les études! («La fac me manque»).

Capacités d'organisation: a de l'expérience. Autonome. Devrait bénéficier de I'allocation de Pôle Emploi durant les trois années.

Capacités relationnelles : bonnes.

Motivations : études de psycho pas assez concrètes (absence de perspective d'emploi). Avait des préjugés sur le métier d'ASS qu'elle a levés avec discussion auprès de 2 ASS. 1 sœur en première année Éduc Spé. 1 sœur qui fait des études de psycho. Mère secrétaire, père médecin (DCD). Attirée par I'histoire et les valeurs de l'école.

Commentaire général : une excellente candidate!

Le mode de recrutement dans les cursus de travail social ne repose alors pas simplement sur une légitimité scolaire, mais bien plus sur une légitimité qu'on pourrait qualifier d'« expérientielle». En témoigne cette "curiosité» très recherchée par les formateurs ${ }^{23}$, qui ne se réduit pas uniquement à la «curiosité intellectuelle» (décrite par Bourdieu \& Passeron, 1970) qui caractérisait les enfants de familles culturellement favorisées et qui renvoyait à la possession des connaissances scolaires et culturelles. Les écoles sociales cherchent des individus qui puissent se conformer aux attentes, qui soient prêts à une "réflexivité», qui puissent travailler en restant dans les cadres de la formation, qui soient "autonomes», tant du point de vue économique que scolaire. Mais la propension à une «distance à soi », à la «réflexivité», à «parler de soi », dépend de la position occupée dans l'espace social (position qui est ici «en cours de formation » et donc spécialement caractérisée par l'incertitude et la redéfinition), et elle est de plus davantage présente chez des individus issus des professions intermédiaires (Poliak, 2002, p.7). L'accent est dès lors mis sur le contact que la candidate peut avoir eu avec le champ du «social», alors que l'éloignement du social, le manque de connaissance de la formation ou du métier peuvent conduire à l'attribution de notes plus moyennes, même pour des candidates ayant un parcours tout à fait « régulier ». C'est notamment le cas de Ségolène, issue d'une famille de classes supérieures et plutôt dotée à tous points de vue ${ }^{24}:$ si on lui recon-

23 "On évalue une entrée en formation... ce qu'on va évaluer c'est la curiosité!». Entretien avec Nicole.

24 Ségolène, 19 ans, bac littéraire à l'heure ; père commissaire de naît des qualités - «a su s'interroger sur ses préjugés face à certaines problématiques -> ébauche de déconstruction [...]; points forts : savoir-faire, ouverture, équilibrée, pas de soucis financiers »-, on lui reproche des connaissances insuffisantes du métier - «n'a pas rencontré d'ASS : connaissance de la formation, mais moins de la profession; connaissance du métier insuffisante ». Elle est notée $12 / 20^{25}$.

Les changements progressifs du public (plus jeune et davantage d'origine populaire) à l'entrée de ces cursus font qu'à l'heure actuelle un grand nombre de candidates ne correspond plus aux attentes des membres du jury ${ }^{26}$. Si les étudiantes des classes supérieures peuvent certes être favorisées par un ensemble de dispositions et de ressources (socio-scolaires et économiques) mobilisables devant les jurys, une excessive aisance et un manque de connaissances sur certaines réalités du métier peuvent néanmoins entraver une bonne réussite aux épreuves. Une étudiante issue des classes supérieures, ou en tout cas davantage dotée en ressources, peut alors induire une plus longue évaluation de la part des formateurs. Ceux-ci peuvent remettre en question ses motivations, redoutant que la candidate puisse être « déçue » par cette courte formation, professionnelle et «intermédiaire», et ne pas poursuivre jusqu'au bout des trois ans, ce que met en valeur aussi S. Orange dans le cas des STS (Orange, 2011). Faute de ces qualités informelles et d'un vécu particulier, les centres de formation «se contentent» alors d'étudiantes qui ont un niveau scolaire «moyen » et qui s'intéressent à l'actualité. Comme nous le souligne le cadre pédagogique d'un autre centre de formation, le «bon candidat» :

c'est quelqu'un qui va être curieux des enseignements qu'il va avoir, qui se sera renseigné en postulant un peu avant des matières qu'il aura, de comment les choses s'organisent, qu'il est allé chercher sur internet en discutant avec les étudiants dans les forums, et c'est quelqu'un qui va se poser des questions et qui ne va pas se censurer, qui va accepter de confronter ses questionnements et ses incertitudes auprès des enseignants et auprès des groupes de pairs et qui est prêt à recevoir une remise en discussion, prêt à être

la marine, mère au foyer (ancienne infirmière à domicile), quatrième d'une fratrie de 8 enfants; BAFA, scouts et activités associatives de «maraude» auprès des sans domicile.

25 Fiche d'évaluation de Ségolène, manuscrite par deux formateurs à la suite de son entretien, dernière session de sélection 2013.

26 Suite aux financements régionaux, l'école ne peut pas trop se permettre de "perdre» des étudiantes au fil des trois ans. 
bousculé pendant les études. Bon, après je sais qu'on est aussi dans un rythme un peu scolaire, donc ici quelqu'un qui a des bonnes notes, qui a appris ses leçons et qui a un bon niveau d'expression et communication... il y a une exigence scolaire avec le double cursus! ${ }^{27}$

Arrêtons-nous maintenant sur le cas des jeunes femmes d'origine supérieure. Nous avons montré qu'elles peuvent se positionner dans l'ensemble différemment des étudiantes d'origine moyenne et populaire vis-à-vis du processus de sélection. C'est alors dans les différentes réappropriations qu'elles font de l'offre de formation et dans les constructions de leurs stratégies professionnelles que l'origine sociale ressort davantage.

\section{Les étudiantes d'origine supérieure : des «héritières" ?}

Tout d'abord, la perception de cette filière peut changer après l'entrée en formation. Un sentiment de décalage face aux autres étudiantes composant la promotion peut porter à vivre la formation de manière différente, à se détacher progressivement de l'ensemble des étudiantes, à chercher ailleurs des réorientations et des engagements. C'est ce que nous raconte Ségolène au sujet de son entrée à l'école :

[...] déjà je suis rentrée et on va dire que $70 \%$ de la population était noire et moi j'étais dans des collèges et lycées privés, où il y avait deux-trois noirs dans l'école et ça se limitait à ça... Et là tu fais «Euh, misère... », et quand tu n'es pas habitué ça fait quand même un choc! [...] y a une différence de mentalité entre nous, je n'ai pas reçu la même éducation que la plupart d'entre eux, y a un décalage pas forcément évident quand... tous les jours à l'école, dans les conversations, on vit pas du tout les mêmes choses... ${ }^{28}$

Cette distance sociale et ethnique par rapport aux autres étudiantes est également soulignée par Margaux ${ }^{29}$, étudiante d'un autre centre de formation

27 Entretien avec Mathilde, 40 ans environ. ASS de formation, elle a travaillé pendant 15 ans entre la polyvalence de secteur et le service social scolaire, pour ensuite devenir responsable «administrative et pédagogique» de la formation d'ASS.

28 Entretien avec Ségolène, avril 2015.

29 Margaux a 22 ans à l'entrée en formation et est issue d'un père (où la proportion d'élèves issus des classes populaires est plus élevée) :

J'ai rencontré des gens mais en fait c'est con à dire, mais au niveau du milieu social je corresponds à rien par rapport à tous les gens qui sont autour de moi et du coup... c'est quelque chose qu'on sent... même dans la façon de s'exprimer, de s'habiller, je vais dire... dans les intérêts... voilà je peux passer $35 \mathrm{~h}$ par semaine avec eux, il faut bien que je m'entende avec quelqu'un, mais dès qu'il y a du débat, des discussions de groupe, je ne peux absolument pas prendre part, ce n'est pas volontaire, c'est juste que je me dis que je ne vais surtout pas intervenir là-dessus [...] j'ai vu la réaction de mon père, de mes parents, quand je leur ai dit que j'allais vers le social, ils m'ont gentiment expliqué que voilà en gros, ça correspondait pas à ma classe sociale, en fait... ${ }^{30}$

Face à ce trop grand sentiment de désajustement, Margaux décide d'ailleurs de quitter ce centre de formation pour se réorienter vers une autre école où elle trouvera des collègues plus proches socialement. Ségolène se maintient quant à elle temporairement à l'écart de sa promo, en s'engageant dans des mouvements religieux. Soulignons toutefois qu'au terme de ses trois années de formation, Ségolène a échoué à l'épreuve finale devant lui délivrer le diplôme.

À cet égard, si le taux de réussite aux épreuves finales du diplôme d'État est relativement élevé (entre 79 et $85 \%$; DREES, 2016), les abandons, réorientations, échecs et redoublements au cours des trois ans ne sont pourtant pas rares. Et, de ce point de vue, les étudiantes des classes supérieures se distinguent tout particulièrement. Sur 21 étudiantes des classes supérieures interrogées au début de l'enquête en 2013, seulement huit sont parvenues jusqu'au diplôme final (auxquelles il faut ajouter deux étudiantes ayant intégré la promotion en cours de route suite à un redoublement), alors que chez les autres étudiantes le taux de réussite est supérieur de dix points (53\% contre $43 \%)$. Quant au sort des treize autres étudiantes, sept quittent la formation au fil des trois années, soit après un échec lors d'épreuves, soit suite à un décrochage progressif en raison d'un rythme de formation trop élevé, soit pour des reconversions dans d'autres filières du supérieur, soit, enfin, pour attendre de passer l'épreuve finale avec une année de maturité supplémentaire; comme Ségolène, quatre étudiantes, ayant accompli les trois années de formation, ne parviennent

habite avec sa sœur, qui mène des études de théâtre.

30 Entretien avec Margaux, octobre 2014. 
pas à passer les épreuves du diplôme d'État, même si l'école avait «approuvé» leur passage ${ }^{31}$.

Sans surestimer ici les appartenances de classes et donner des raisons «collectives» aux réorientations, changements d'établissement ou échecs, ces décalages nous semblent renvoyer tant au poids des différences d'âge qu'à celui des héritages familiaux. De ce point de vue, porter attention à la pluralité de ces héritages peut aider à mieux saisir les différentes ressources de ce public pour faire face à la formation. D'abord, si l'on s'attache à regarder la composition des fratries de ces étudiantes de classes supérieures, on trouve parmi celles-ci un pourcentage plus important d'aînées (31 \% contre 22 \%) dont les frères et sœurs suivent des études supérieures ou travaillent en tant que cadres ( $49 \%$ contre $31 \%$ ). Elles sont également les plus soutenues financièrement par leurs parents pendant la première année de formation (71 \% contre $57 \%)$ et vivent plus souvent hors du domicile parental (61\% contre $55 \%$ ). Par ailleurs, ces étudiantes sont plus nombreuses à avoir eu une expérience professionnelle avant d'intégrer l'école (86 \% contre $76 \%$ ), régulièrement à des postes qui leur ont permis d'acquérir un ensemble de savoir-faire et savoir-être susceptibles d'être reconvertis dans ce secteur d'emploi (le babysitting ou l'animation par exemple).

De plus, I'héritage familial socio-culturel ne coïncide pas forcément avec un héritage purement «scolaire» et il convient par conséquent de distinguer les parents cadres "populaires ascendants» et les "héritiers» (Peugny, 2007, p. 205-216). Les pères «cadres» des étudiantes de notre échantillon sont dans une large mesure diplômés du supérieur (80 \% contre $37 \%$ des pères de professions intermédiaires et $11 \%$ des pères de catégories populaires). Ils travaillent plus souvent dans le privé ou en profession libérale que dans le public (23\%). L'homogamie concerne une minorité de parents au sein de ce public $(\mathrm{N}=10)$, et principalement des parents médecins $(\mathrm{N}=4)$. Ces pères «cadres « $^{32}$ sont majoritairement d'origine moyenne ou populaire ( $N=19$ ) et dans une moindre mesure d'origine plus favorisée $(N=11)$. Du côté maternel, le poids des grands-pères «cadres » suit la même dynamique (11 sur 32 répondants chez les étudiantes de classes supé-

31 C'est effectivement le centre de formation, en fin de troisième année, qui décide, en concertation avec ses étudiantes, lesquelles présenter aux épreuves régionales pour l'obtention du titre. 32 Les questions concernant les grands-parents connaissent un important taux de non-réponse, de l'ordre de $43 \%$. rieures). Ces résultats donnent à voir une trajectoire ascendante familiale qui s'arrête souvent aux parents («cadres populaires ascendants» plutôt qu'« héritiers»).

Cette population d'étudiantes de classes supérieures ne constitue donc pas une élite $d$ 'héritières fortement dotées en ressources économiques, sociales, scolaires et symboliques depuis plusieurs générations. Leur orientation dans le travail social doit moins être lue comme le résultat d'un déclassement que comme celui d'un reclassement familial. Ce choix a priori «improbable» relève en effet plus d'un déclin social «relatif» pour ces femmes de classes supérieures. Assistante sociale reste un métier envisageable par une partie de ces classes supérieures en recomposition, a fortiori dans la mesure où il donne accès à une stabilité dans la fonction publique, s'éloignant en cela d'autres «métiers féminins» moins réputés tels par exemple que celui d'aide à domicile (Avril, 2014) et caractérisés par une carrière moins stable, plus soumise à l'incertitude statutaire et contractuelle.

Le cas d'Emma ${ }^{33}$, sus-citée, considérée par ses formateurs comme une des meilleures élèves de la promotion, va nous permettre de mieux rendre compte de ces trajectoires particulières, en articulant statistiques et récits de vie (Pagis, 2011). Emma n'intègre pas la formation d'assistante sociale directement après l'obtention de son baccalauréat, mais à 26 ans. Après une licence en psychologie, elle ne poursuit pas ces études car elle appréhende les débouchés de la formation. Elle décide alors de recommencer des études de lettres à I'université et dans une école de journalisme qu'elle finit par abandonner, car elle «n'apprécie pas le milieu ». Elle enchaîne alors les petits boulots (baby-sitter, serveuse et vendeuse), dans une période qu'elle décrit comme étant marquée par une forte confusion. Elle finit par intégrer un travail d'adjoint administratif dans l'action sociale communale de sa ville :

Je me suis mise à travailler et j'ai travaillé un an et demi pour la mairie de ma ville dans le secteur du social pour les personnes âgées et handicapées. Au début j'étais juste chargée de faire les archives et vu que j'ai fini ce boulot-là très rapidement, je suis montée en grade et j'ai instruit des dossiers d'aide sociale, d'APA [allocation personnalisée d'autonomie], de PCH [prestation de compensation du handicap], d'aide à

33 Loin de représenter un cas anecdotique, Emma est le premier «parangon » (les parangons étant les cinq individus les plus représentatifs pour chaque classe fournis par le logiciel) du troisième groupe de la classification présentée plus haut. 
domicile et ça m'a vraiment plu... C'était vacations sur vacations... et là je me suis dit : j'ai envie de travailler dans le social, faire un travail qui a du sens, me lever le matin et faire quelque chose pour laquelle je suis motivée. Et donc voilà. Malheureusement je n'ai pas pu être titularisée là-bas et du coup [...] je me suis présentée à l'école. [...] Tout le monde était super content que je reprenne des études! [...] À part mon copain qui est thésard, la plupart ils travaillent, sont tous bac $+5,+8$..

Elle révise les mois précédant les épreuves de sélection avec l'aide de son copain et de la mère de celui-ci. Une fois passé l'examen d'entrée, elle déclare qu'elle s'attendait ce que ce soit plus compliqué. Elle intègre l'école et se maintient à 15 de moyenne durant les trois années, parvenant à «s'en sortir sans bosser». Elle obtient le diplôme d'État sans grandes difficultés au terme des trois ans. Après les épreuves finales, elle rejoint son compagnon (devenu son mari) en Amérique, où il a trouvé un poste temporaire dans le milieu de la recherche académique. Elle suspend pour l'instant son projet d'exercer dans le social.

Ainsi, si l'orientation dans ces filières peut représenter pour les plus jeunes une manière d'échapper aux stratégies de classement scolaire (l'admission postbac [APB] par exemple) en visant des formations professionnelles courtes, pour Emma comme pour d'autres étudiantes plus âgées, cette formation peut aussi représenter une manière de se reclasser après des phases d'incertitude. Le «choix» du métier d'assistante sociale représente un épisode "temporaire», qui permet l'obtention d'un diplôme professionnel dans une trajectoire scolaire et professionnelle relativement incertaine.

\section{Conclusion}

Une simple explication en termes de déclassement pour expliquer l'orientation de femmes de classes supérieures dans la formation d'assistante sociale paraît clairement insatisfaisante. Il serait également erroné de I'interpréter sur la base d'une naturalisation de compétences «positives» de "dévouement aux autres». De surcroît, tandis que le choix des étudiantes des classes supérieures pourrait sembler à première vue «improbable», nous avons aussi mis en évidence combien les stratégies des écoles influent sur les parcours étudiants, en exigeant d'une part des candidats des propriétés intellectuelles et économiques spécifiques (Maurel, 2015) et en mettant l'accent, au moment du recrute- socialement situées, en conformité avec les valeurs «petites bourgeoises» de «respectabilité » et de «curiosité » (Bourdieu, 1979; Gombert \& van Zanten, 2004; Skeggs, 2015).

Ces choix d'orientation et de reconversion doivent alors être interprétés en lien avec I'héritage social et familial, les dynamiques d'âges et les évolutions de l'offre de formation, au croisement des aspirations produites par le système d'enseignement et des chances effectives de ces étudiantes (Bourdieu, 1978). Le choix de ce cursus pour ces jeunes femmes n'ayant pas suivi un chemin scolaire balisé doit donc être considéré comme une manière de concilier la poursuite d'études supérieures, l'arrivée certaine et rapide dans un métier où le chômage est très faible, et le respect de certaines traditions morales familiales, notamment pour celles qui ont reçu une éducation catholique.

Loin de représenter des cursus «atypiques», ces formations, par leurs caractéristiques intrinsèques (des études courtes, professionnelles, mélangeant des orientations à la frontière de plusieurs disciplines académiques : psychologie, sociologie, philosophie, sciences de l'éducation) et extrinsèques (des métiers qui mobilisent des dispositions «relationnelles», permettant l'accès à une position d'entre-deux «qualifiée», entre encadrement administratif et "souci des autres»), peuvent représenter un «bon compromis» pour une partie de ces étudiantes en reclassement social et scolaire. Si elles reflètent l'injonction à la professionnalisation fortement présente aujourd'hui lors des choix scolaires, elles permettent, entre vocation et choix rationnel, entre déterminisme et rationalisme, de se reconvertir tout en se protégeant du chômage.

Ruggero lori Université de Versailles-Saint-Quentin-en-Yvelines, laboratoire PRINTEMPS iori.ruggero@gmail.com

\section{Bibliographie}

AGULHON C. (2005). «Les politiques régionales de formation professionnelle. Du référentiel commun à la politique locale». Éducation et sociétés, ${ }^{\circ} 16$, p.279-292.

ASTIER I. (2009), «Les transformations de la relation d'aide dans l'intervention sociale ». Informations sociales, $\mathrm{n}^{\circ} 152$, vol.2, p. 52-58.

AVRIL C. (2014). Les aides à domicile : un autre monde populaire. Paris : La Dispute.

BLANCHARD M. (2012). «S'orienter en école de commerce : goût de l'utile ou choix du raisonnable?». Sociologies, 
$\mathrm{n}^{\circ} 15$. En ligne : <http://sociologies.revues.org/4077> (consulté le 10 décembre 2015).

BODIN R. (2009). "Les signes de l'élection». Actes de la recherche en sciences sociales, $\mathrm{n}^{\circ} 178$, vol.3, p. 80-87.

BOURDIEU P. (1974). «Avenir de classe et causalité du probable». Revue française de sociologie, vol.15, n 15, p.3-42.

BOURDIEU P. (1978). «Classement déclassement, reclassement ». Actes de la recherche en sciences sociales, $\mathrm{n}^{\circ} 24$, vol.1, p. 2-22.

BOURDIEU P. (1979). La Distinction. Critique sociale du jugement. Paris : Éd. de Minuit.

BOURDIEU P. (dir.) (1993). La misère du monde. Paris : Éd. du Seuil.

BOURDIEU P. \& de SAINT-MARTIN M. (1970). «L'excellence scolaire et les valeurs du système d'enseignement français ». Annales, n¹, vol.25, p.147-175.

BOURDIEU P. \& PASSERON J.-C. (1964). Les Héritiers. Les Étudiants et la Culture. Paris : Éd. de Minuit.

BOURDIEU P. \& PASSERON J.-C. (1970). La Reproduction. Paris : Éd. de Minuit.

BRAMS L. \& COURTECUISSE N. (1972). Les assistantes de service social. Contribution à la sociologie d'une profession. Paris : INSERM.

CHARLE C. \& SOULIÉ C. (2008). Les ravages de la «modernisation » universitaire en Europe. Paris : Syllepse.

CONVERT B. (2003). «Des hiérarchies maintenues». Actes de la recherche en sciences sociales, $\mathrm{n}^{\circ} 49$, vol.4, p.61-73.

CONVERT B. (2010). «Espace de l'enseignement supérieur et stratégies étudiantes». Actes de la recherche en sciences sociales, $n^{\circ} 183$, vol.3, p. 14-31.

CRAPUCHET S. (1987). «Qui étaient-elles et qui sont-elles? 1905-1976». Vie sociale, n 8-9, p. 417-443.

DARMON M. (2013). Les classes préparatoires. La fabrique d'une jeunesse dominante. Paris : La Découverte.

DELAUNAY B. (2006). «Évolution d'un savoir de référence en service social : la psychologie». In M. Bresson (dir.), La psychologisation de l'intervention sociale : mythes et réalités. Paris : L'Harmattan, p. 177-189.

DIRECTION DE LA RECHERCHE, DES ÉTUDES, DE L'ÉVALUATION ET DES STATISTIQUES [DREES] (2015-2016). L'enquête annuelle sur les écoles de formation aux professions sociales. En ligne : <http://drees.solidarites-sante.gouv.fr/etudes -et-statistiques/open-data/professions-de-sante-et-dusocial/article/l-enquete-annuelle-sur-les-ecoles-deformation-aux-professions-sociales> (consulté le 10 décembre 2015).

DUBOIS V. (2013). La culture comme vocation. Paris : Raison d'Agir.

ECKERT H. (2014). «Déclassement et hantise du déclassement ». Revue française de pédagogie, n¹88, p. 87-108.

GASPAR J.-F. (2007). «Entreprise de légitimation et de délégitimation des savoirs scolaires et "de terrain" dans l'univers du travail social ». Cahiers de la recherche sur l'éducation et les savoirs, n6, p. 153-169.
GOMBERT P. \& van ZANTEN A. (2004). «Le modèle éducatif du pôle "privé" des classes moyennes : ancrage et traduction dans la banlieue parisienne». Éducation et sociétés, $\mathrm{n}^{\circ} 14$, p. 67-83.

GUERRAND R.-H. \& RUPP M.-A. (1978). Brève histoire du service social en France (1896-1976). Toulouse : Privat.

HENRI-PANABIÈRE G. (2010). Des «héritiers » en échec scolaire. Paris : La Dispute.

HUGRÉE C. (2010). «Le CAPES ou rien?». Actes de la recherche en sciences sociales, $\mathrm{n}^{\circ} 183$, p.72-85.

HUGRÉE C. \& POULLAOUEC T. (2011). «Qui sont les étudiants d'origine populaire?». In O. Galland, E. Verley \& R. Vourc'h, Les mondes étudiants. Enquête Conditions de Vie 2010. Paris : La Documentation française, p. 15-26.

IORI R. (2017). «Entre universitarisation et "professionnalisation" : la formation des assistant-e-s de service social en France et en Italie». Formation Emploi, n 138.

IORI R. \& NICOURD S. (2014). «La construction des discours vocationnels comme support de légitimité dans le champ de l'intervention sociale». Vie sociale, $n^{\circ} 8$, vol.4, p.101-112.

LAHIRE B. (1994), «Les raisons de l'improbable : les formes populaires de la "réussite" à l'école élémentaire». In V. Guy (dir.), L'Éducation prisonnière de la forme scolaire? Scolarisation et socialisation dans les sociétés industrielles. Lyon : Presses universitaires de Lyon, p.73-106.

MAUREL A. (2015). "Quels sont les déterminants des choix d'orientations dans l'enseignement supérieur». Regards croisés sur l'économie, ${ }^{\circ}{ }^{16}$, vol.1, p. 69-79.

MERCKLÉ P. (2005). «Une sociologie des irrégularités sociales est-elle possible? ». Idées, la revue des sciences économiques et sociales, $\mathrm{n}^{\circ} 142$, p. $22-29$

MOLINA Y. (2016). «L'accès aux formations sociales entre choix d'orientation professionnelle et stratégies». Formation Emploi, nº132, p.117-137.

MUEL-DREYFUS F. (1983). Le métier d'éducateur. Paris : Éd. de Minuit.

NEYRAT F. (2008). «Le travail à l'épreuve de la compétence». Savoir/Agir, n³, vol.1, p.31-36.

NICOURD S., SAMUEL O. \& VILTER S. (2011). «Les inégalités territoriales à l'université : effets sur les parcours des étudiants d'origine populaire». Revue française de pédagogie, $\mathrm{n}^{\circ} 176$, p. 27-40.

ORANGE S. (2011). L'autre supérieur. Aspirations et sens des limites des étudiants de BTS. Thèse de doctorat, sociologie, université de Poitiers.

OBSERVATOIRE NATIONAL DE LA VIE ÉTUDIANTE [OVE] (2013). Repères Enquête Conditions de vie. Paris : Observatoire national de la vie étudiante. En ligne : <http://www. ove-national.education.fr/l-enquete/2013> (consulté le 10 décembre 2015).

PAGIS J. (2011). «Engagements soixante-huitards sous les regards croisés des statistiques et des récits de vie». Politix, n 93, p. 93-114.

PAGIS J. \& PASQUALI P. (2016), «Observer les mobilités 
sociales en train de se faire. Micro-contextes, expériences vécues et incidences socio-politiques ». Politix, $\mathrm{n}^{\circ} 114$, p.7-20.

PALHETA U. (2011). «Enseignement professionnel et classes populaires : comment s'orientent les élèves "orientés"». Revue française de pédagogie, $n^{\circ} 175$, p. 59-72.

PASQUALI P. (2014). Passer les frontières sociales. Paris : Fayard.

PEUGNY C. (2007). La mobilité sociale descendante. L'épreuve du déclassement. Thèse de doctorat, sociologie, Institut d'études politiques de Paris.

POLIAK C. (2002). "Manières profanes de "parler de soi"». Genèses, n 47 , p. 4-20.

PROST A. (2013). Du changement dans l'école. Les réformes de l'éducation de 1936 à nos jours. Paris : Éd. du Seuil.

ROPÉ E. \& TANGUY L. (1994). Savoirs et compétences : de l'usage de ces notions dans l'école et l'entreprise. Paris : L'Harmattan.
SKEGGS B. (2015). Des femmes respectables. Marseille: Agone.

TRALONGO S. (2008). «La méthode du "Projet personnel et professionnel" : un "travail de soi" des étudiants». Sociologies pratiques, $n^{\circ} 17$, p. 95-105.

TRUONG F. (2015). Jeunesses françaises. Bac +5 made in banlieue. Paris : La Découverte.

UNION NATIONALE DES ASSOCIATIONS DE FORMATION ET DE RECHERCHE EN INTERVENTION SOCIALE [UNAFORIS] (2014). L'attractivité des formations de niveau III. État des lieux et perspectives. Commission permanente niveau III, juin 2014 (non publié).

VERDÈS-LEROUX J. (1978). Le travail social. Paris : Éd. de Minuit.

VOZARI A.-S. (2014). "Recruter des "bonnes" assistantes maternelles. La sélection à l'entrée d'un emploi féminin non qualifié». Sociétés contemporaines, n95, vol.3, p.29-54. 